\title{
ESTUDO PROSPECTIVO DE 100 CASOS DE “CORE" BIÓPSIA DIRIGIDA POR ULTRA-SOM E REVISÃO DA LITERATURA*
}

\author{
Ana Lúcia Kefalás Oliveira Melo ${ }^{1}$, Maria Fernanda Curi Barra ${ }^{2}$, Andreza Vargas da Silva ${ }^{2}$, \\ Mariana Kefalás Oliveira ${ }^{3}$, Délcio Scandiuzzi ${ }^{4}$, Antônio Carlos Menezes ${ }^{5}$
}

Resumo OBJETIVO: Mostrar a eficácia do método para evitar biópsias excisionais, verificar suas dificuldades técnicas, definir entre seguimento e biópsia excisional nos nódulos categoria IV do BI-RADS ${ }^{\mathrm{TM}}$ e agilizar o procedimento cirúrgico nos casos de nódulos altamente suspeitos de malignidade (categoria V). MATERIAIS E MÉTODOS: As pacientes foram submetidas a exame clínico de rotina, mamografia e ultra-som. A "core" foi feita com pistola automática e agulha número 14, e foram colhidas de quatro a oito amostras. RESULTADOS: Das 100 lesões estudadas, 47 foram submetidas à cirurgia, além da "core", e diagnosticaram-se 34 carcinomas $(34,0 \%)$. Das 23 lesões classificadas como categoria III, identificou-se apenas um carcinoma $(4,34 \%)$; das 43 classificadas como categoria IV, sete $(16,28 \%)$; e das 34 classificadas como categoria V, $26(76,47 \%)$. Evitou-se a biópsia excisional em 53 casos $(53,0 \%)$. Identificou-se dificuldade no método da "core" em sete casos $(7,0 \%)$, devido a material insuficiente, risco para malignidade envolvendo lesões esclerosantes complexas e discordância entre imagem e histologia. As 33 lesões com resultados de malignidade à punção biópsia por agulha grossa foram confirmadas após a biópsia cirúrgica. Em um caso o diagnóstico pela "core" foi de hiperplasia ductal atípica e após a biópsia cirúrgica da peça diagnosticou-se carcinoma, correspondendo a um resultado falso-negativo. Não houve nenhum resultado falso-positivo. Unitermos: Doenças mamárias - diagnóstico; Neoplasias mamárias; Biópsia por agulha - métodos; Ultrasonografia mamária.

Abstract Prospective study of 100 patients submitted to ultrasound-guided core biopsy and review of the literature. OBJECTIVE: To demonstrate the efficacy of core-needle biopsy to avoid unnecessary surgery; to assess the technical difficulties of this method; to establish decision criteria for clinical follow-up or surgery in patients with lesions classified as suspicious for malignancy (BI-RADS IV); to expedite surgery in patients with lesions classified as highly suspicious for malignancy (BI-RADS V). MATERIALS AND METHODS: The patients were submitted to clinical examination, mammography and ultrasound. Core-needle biopsy was done using a 14-gauge needle, and 4-8 cores per lesion were obtained. RESULTS: A hundred lesions were studied, 47 of them underwent surgery and biopsy, and 34 cancers were diagnosed. In 23 lesions classified as probably benign (III) only one case of cancer was diagnosed (4.34\%). In 43 lesions classified as IV, seven (16.28\%) cancers were found whereas in 34 lesions classified as V, $26(76.47 \%)$ cases of cancer were identified. It was possible to avoid surgery in 53 cases $(53 \%)$. Difficulties in core-needle biopsy occurred in seven cases including insufficient material, risk of malignancy due to sclerosing lesions and lack of correlation between imaging and core-needle biopsy results. All the core biopsy diagnoses of malignancy were confirmed by surgery. There was one false negative result when the core biopsy specimen examination showed atypical hyperplasia while surgery specimen analysis revealed a carcinoma. There were no false positive cases.

Key words: Breast disease - diagnosis; Breast cancer; Core-needle biopsy - methods; Breast ultrasound.

\section{INTRODUÇÃO}

A biópsia cirúrgica de mama foi, por um longo período, o método mais usado para

* Trabalho realizado no Departamento de Clínica Médica, Disciplina de Diagnóstico por Imagem, da Faculdade de Medicina do Triângulo Mineiro (FMTM), Uberaba, MG.

1. Professora Adjunta, Chefe da Disciplina de Diagnóstico por Imagem da FMTM.

2. Acadêmicas do Curso de Medicina da FMTM.

3. Médica Residente do Serviço de Ginecologia e Obstetrícia da Faculdade de Medicina de Ribeirão Preto da Universidade de São Paulo.

4. Médico Mastologista da FMTM.

5. Médico Patologista do Hospital Dr. Hélio Angotti.

Endereço para correspondência: Profa. Dra. Ana Lúcia Kefalás de Oliveira Melo. Rua Santo Antônio, 71. Uberaba, MG, 38010-160. E-mail: frmelo@terra.com.br

Recebido para publicação em 20/5/2003. Aceito, após revisão, em 3/6/2003. determinar a benignidade ou malignidade das anormalidades clínicas ou mamográficas. Todavia, é mais dispendiosa, carrega consigo certa morbidade e deixa cicatrizes. A punção biópsia aspirativa com agulha fina (PBAAF) e a "core" biópsia são, na atualidade, técnicas minimamente invasivas altamente difundidas. Mais recentemente, introduziu-se a mamotomia, técnica capaz de adquirir volumes maiores do tecido mamário, porém de maior custo.

A "core" biópsia tem sido utilizada em larga escala por permitir a obtenção de resultado histológico, e devido ao fato de a PBAAF proporcionar maior incidência de material insuficiente. Neste procedimento, obtém-se um fragmento que representa uma amostra definitiva da lesão mamária. Se o diagnóstico for de benignidade, a cirurgia pode ser evitada, uma vez que é completamente confiável. É de extrema importância a correlação entre os achados clínicos e os de imagem na resolução da conduta definitiva. Já nos casos de malignidade, a "core" proporciona um resultado definitivo. Mesmo assim, as limitações quanto à sua indicação surgem nas suspeitas de cicatriz radial e na diferenciação do carcinoma in situ puro do microinvasivo. O método está, por exemplo, contra-indicado nos casos de coagulopatias, na maioria das pacientes com próteses mamárias ou 
em lesões muito pequenas, próximas à musculatura peitoral ou muito superficiais.

Os objetivos deste estudo prospectivo foram: mostrar a eficácia do método de "core" biópsia em evitar biópsias excisionais; verificar suas dificuldades técnicas; definir entre seguimento clínico e radiológico ou biópsia excisional nos nódulos com classificação IV do Breast Imaging Reporting and Data System (BI-RADS ${ }^{\mathrm{TM}}$ ), e agilizar o procedimento cirúrgico nos casos de nódulos altamente suspeitos de malignidade (categoria V).

\section{MATERIAIS E MÉTODOS}

As biópsias foram realizadas em lesões palpáveis e não-palpáveis, e para isto usouse agulha 14 com pistola Bard Corington $\mathrm{Ga}$, com 2,2 cm de excursão. Nesse procedimento, realizado por especialista experiente no método, foram retirados, no mínimo, quatro fragmentos, variando de quatro a oito. As pacientes, além de responderem a um questionário informativo incluindo perguntas sobre uso de medicamentos, alergias e distúrbios de coagulação, assinaram um documento consentindo a realização do procedimento. Os aparelhos de ultra-som utilizados foram o Diasonics GE e o ATL-HDI 1500, e os transdutores, de 10 a $12 \mathrm{MHz}$.

Todas as pacientes com idade superior a 35 anos realizaram mamografia e ultrasonografia antes da "core" biópsia, e aquelas abaixo de 35 anos realizaram, na maioria dos casos, apenas ultra-sonografia mamária. Em primeira instância, todas foram examinadas clinicamente por mastologistas, mediante inspeção e palpação bilateral das mamas.

As lesões incluídas neste trabalho foram: nódulos com ou sem microcalcificações, densidades assimétricas focais, densidades assimétricas com calcificações no seu interior, e distorções arquiteturais do parênquima, todas elas ecograficamente visíveis. Vale lembrar que se excluíram do estudo os agrupamentos de microcalcificações sem nódulos.

As lesões acima descritas foram enquadradas nas categorias III, IV e V do BIRADS. No grupo III (provavelmente benigno) incluíram-se os nódulos sólidos, circunscritos, redondos ou ovalados. No gru- po IV (suspeitos de malignidade), os nódulos sólidos, redondos, ovalados ou macrolobulados com calcificações associadas e/ou margens obscuras em mais de $25 \%$ de sua extensão, as lesões císticas com projeções murais ou septações grossas e os nódulos ecograficamente suspeitos, dentre eles, aqueles com diâmetro ântero-posterior maior que o lateral e/ou com margem obscura angular, ramificada ou mal delimitada e/ou com sombra acústica posterior (atenuação). No grupo V (altamente suspeito de malignidade) inseriram-se os nódulos espiculados com ou sem microcalcificações e as distorções irregulares ou densidades assimétricas focais palpáveis e/ou ultra-sonograficamente suspeitas. Para a mensuração das lesões foi usado seu maior diâmetro. Nas lesões com microcalcificações no seu interior, radiografaram-se os espécimes com ampliação para averiguar a presença das microcalcificações após a realização da biópsia percutânea com agulha grossa (casos das Figuras 1 e 2).

De julho de 1998 a julho de 2001 foram selecionadas, prospectivamente, 100 lesões em 99 pacientes, nas quais se realizou a "core" biópsia guiada por ultra-som, sendo 37 palpáveis e 63 não-palpáveis. Em 47 delas realizou-se a biópsia excisional após o resultado da "core" biópsia. As 53

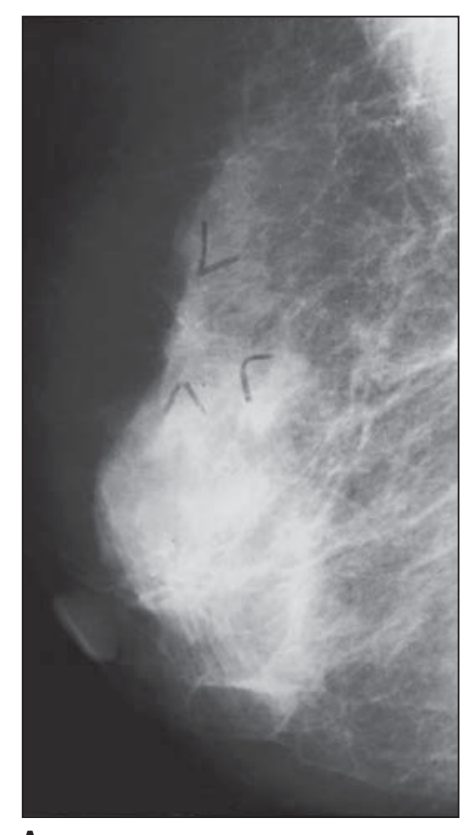

A

Figura 1. Mamografia oblíqua (A) mostrando nódulo irregular, espiculado, de média radiodensidade, com microcalcificações no seu interior, de $1 \mathrm{~cm}$. Ultra-som (B) demonstrando nódulo hipoecóico, heterogêneo,

lesões restantes foram seguidas anualmente e, no caso de aumento de tamanho da lesão, foi realizada cirurgia excisional com marcação pré-cirúrgica ou nova "core" biópsia. Observou-se que, até o momento, houve um caso falso-negativo.

\section{RESULTADOS}

Um total de 100 lesões foi incluído neste estudo, sendo 64 nódulos, 16 nódulos com microcalcificações, 19 densidades assimétricas e uma distorção arquitetural. Antes da "core" biópsia as lesões foram classificadas de acordo com as categorias do BI-RADS (Tabela 1 e Gráfico 1). Podese notar a correlação entre os achados histológicos pela "core" e a classificação do BI-RADS na Tabela 2.

Das 47 lesões submetidas a excisão cirúrgica, $33(72,34 \%)$ foram diagnosticadas

Tabela 1 Distribuição das lesões na classificação do BI-RADS, de acordo com o achado radiológico.

\begin{tabular}{|l|r|r|r|}
\hline \multicolumn{1}{|c|}{ Achado radiológico } & III & IV & V \\
\hline Nódulos & 21 & 28 & 15 \\
Nódulos com microcalcificações & 1 & 3 & 12 \\
Densificações assimétricas & 1 & 11 & 7 \\
Distorção arquitetural & 0 & 1 & 0 \\
\hline Total de lesões & 23 & 43 & 34 \\
\hline
\end{tabular}

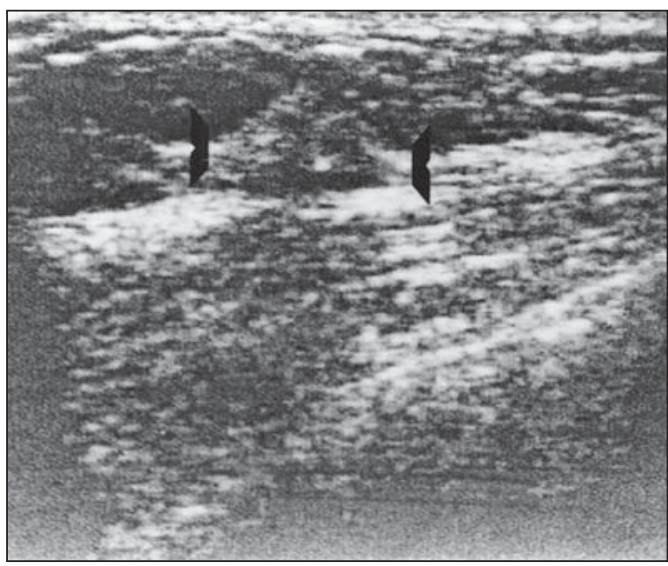
B

irregular, com margem angular e halo ecogênico de 1,1cm.

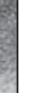

irregular, com margem angular e halo ecogênico de 1,1cm. 


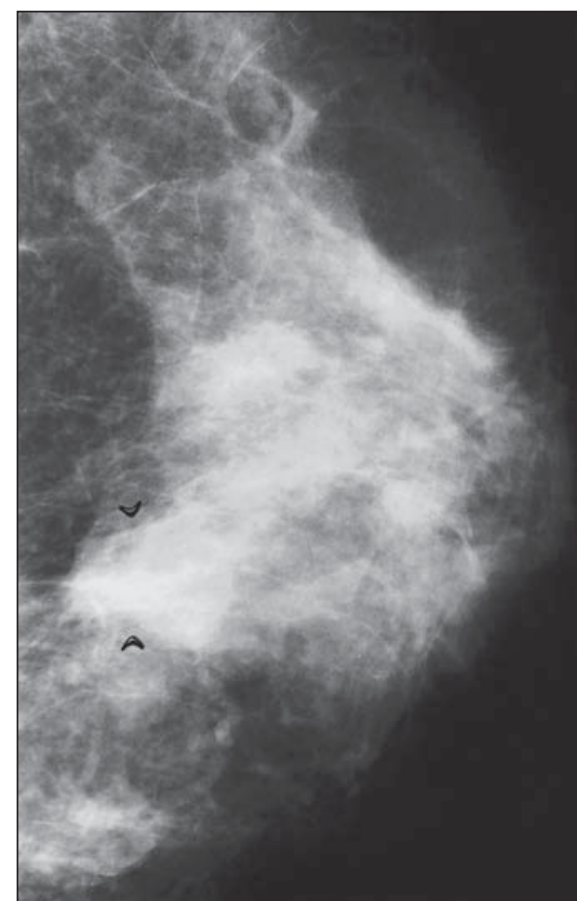

A

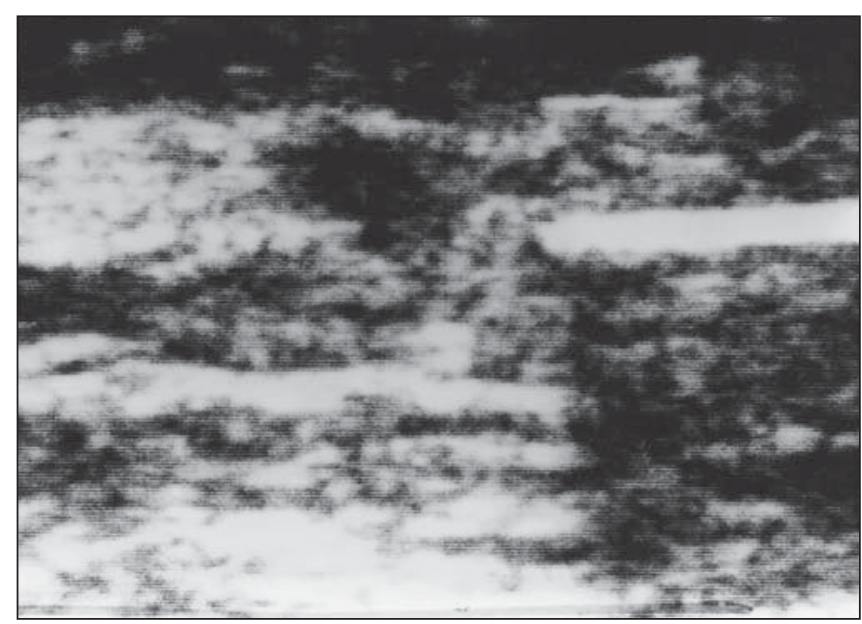

D

Figura 2. Mamografias crânio-caudal (A) e oblíqua (B) mostrando densidade assimétrica, irregular, mal-delimitada, com microcalcificações no seu interior (B). Ampliação (C) com "spot" focal evidenciando melhor a densidade assimétrica. Ultra-sonografia com biópsia dirigida pré (D) e pós-disparo (E) mostrando nódulo hipoecóico heterogêneo, espiculado, com padrão ramificado.

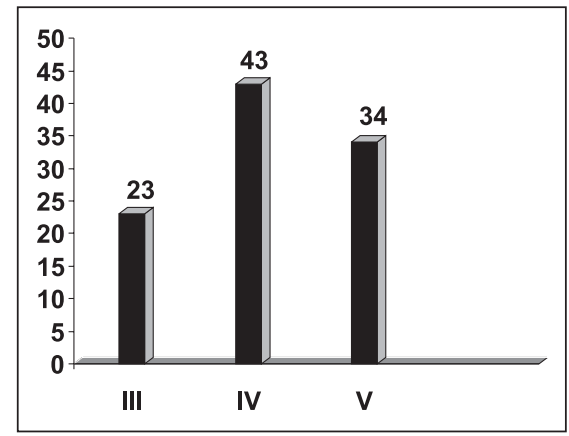

Gráfico 1. Classificação das lesões de acordo com as categorias do BI-RADS.
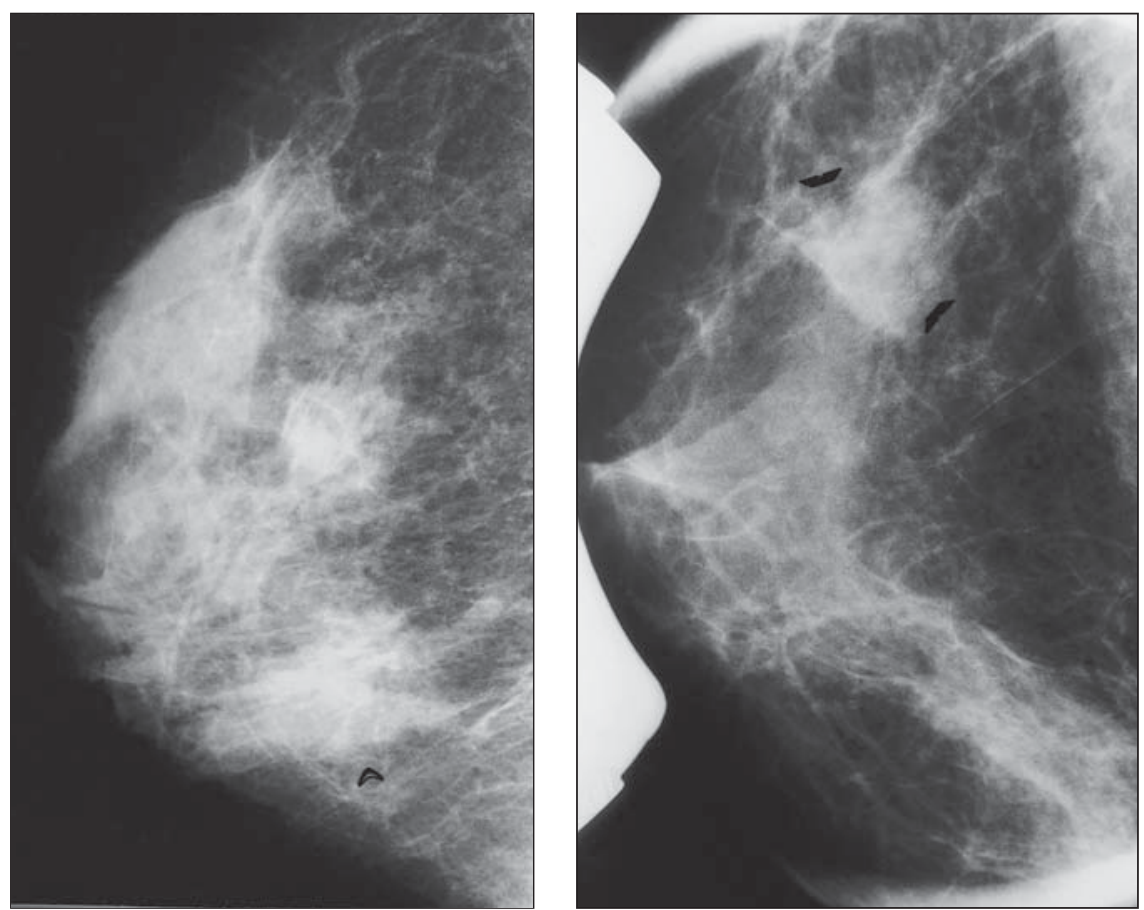

C

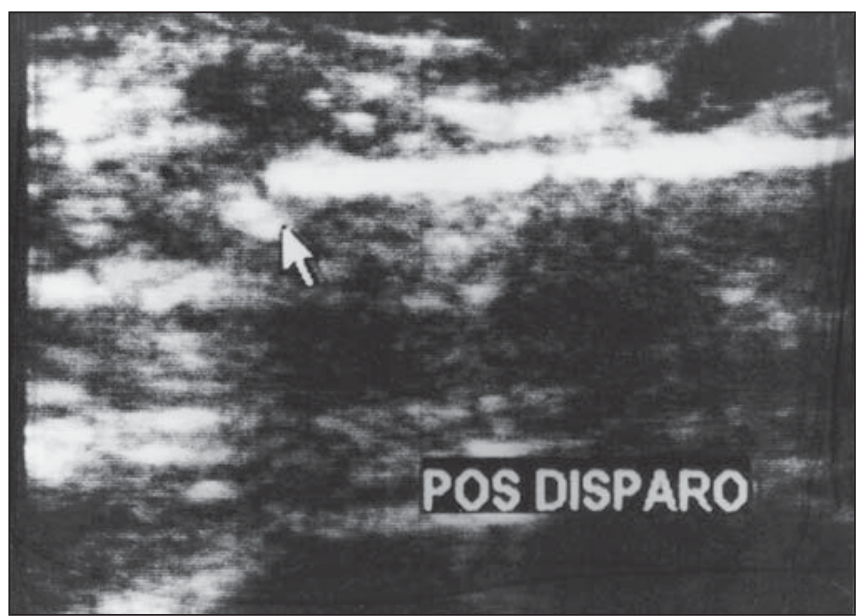

E como carcinoma, de acordo com a "core". Uma lesão teve o diagnóstico de malignidade somente após sua biópsia excisional, uma vez que, pela "core", o diagnóstico foi de hiperplasia ductal com atipia. Outras seis $(12,76 \%)$ foram submetidas a cirurgia por apresentarem resultados de atipia celular (uma lesão), esclerose radial (duas lesões), adenose esclerosante (duas lesões) e material insuficiente (uma lesão). As sete biópsias excisionais restantes aconteceram por indicação do cirurgião ou devido a dis- cordância entre os achados de imagem e o resultado histopatológico da "core".

Nos 34 casos diagnosticados como malignos distribuídos nas categorias III, IV e V (Gráficos 2 e 3) encontraram-se um carcinoma ductal in situ, 29 carcinomas ductais invasivos clássicos, um caso de carcinoma ductal invasivo com componente in situ de pequeno grau, dois carcinomas mucinosos e um carcinoma multifocal. Houve concordância entre os resultados da "core" biópsia e da cirurgia subseqüente 
Tabela 2 Distribuição dos achados histológicos com a "core": correlação com a classificação do BI-RADS.

\begin{tabular}{|l|r|r|r|r|}
\hline \multicolumn{1}{|c|}{ Histopatologia } & III & IV & V & Total \\
\hline Fibroadenoma & 11 & 10 & 0 & 21 \\
Alteração fibrocística & 6 & 5 & 2 & 13 \\
Necrose gordurosa & 1 & 2 & 1 & 4 \\
Lesão esclerosante radial & 0 & 1 & 1 & 2 \\
Adenose esclerosante & 0 & 0 & 2 & 2 \\
Hiperplasia ductal sem atipia & 0 & 3 & 0 & 3 \\
Hiperplasia ductal com atipia & 0 & 1 & 0 & 1 \\
Alteração fibrocística com fibroesclerose & 4 & 12 & 2 & 18 \\
Mastite crônica & 0 & 1 & 0 & 1 \\
Carcinoma & 1 & 7 & 26 & 34 \\
Material insuficiente* & 0 & 1 & 0 & 1 \\
\hline Total & 23 & 43 & 34 & 100 \\
\hline
\end{tabular}

* Diagnóstico após biópsia: papiloma intracístico.

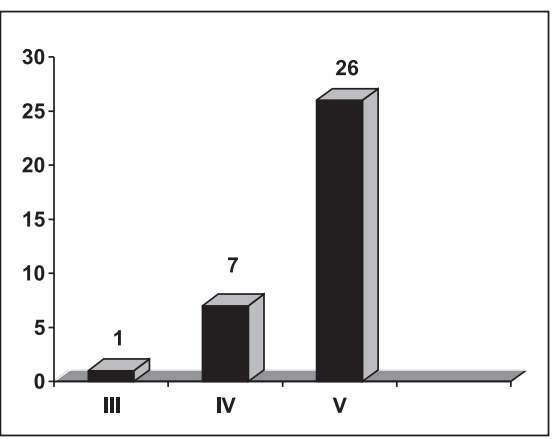

Gráfico 2. Relação entre o diagnóstico de carcinoma e categorias do BI-RADS. em $33(97,06 \%)$ dos 34 casos diagnosticados como carcinoma. Em um caso em que a "core" biópsia demonstrou hiperplasia ductal com atipia, diagnosticou-se carcinoma após a cirurgia. Esta não foi realizada em seis pacientes com categoria $\mathrm{V}$ do BIRADS e em 28 casos da categoria IV, pois nesses casos a "core" biópsia foi considerada definitivamente benigna, havendo também concordância com os achados mamográficos e, assim, o seguimento foi iniciado seis meses após o procedimento da "core" biópsia.

Ressalta-se que a sensibilidade e a especificidade da "core" foram determinadas pelos resultados obtidos com a biópsia cirúrgica e/ou "follow-up" com mamografia para determinar achados falso-positivos e falso-negativos. Neste trabalho não houve nenhum caso de falso-positivo e apenas um caso de falso-negativo. Dessa forma, o valor preditivo positivo foi de $100 \%$ e o valor preditivo negativo, de $92,8 \%$. A sensibilidade e a especificidade foram, respectivamente, de $97 \%$ e $100 \%$.

\section{DISCUSSÃO}

O ultra-som mamário ganhou um importante papel na propedêutica das lesões da mama, não só como adjuvante à mamografia para diferenciação entre lesões císticas e sólidas, mas também nas intervenções mamárias, como método para guiar as biópsias percutâneas.

As biópsias guiadas por ultra-som são obtidas com maior facilidade, uma vez que o equipamento é economicamente viável e

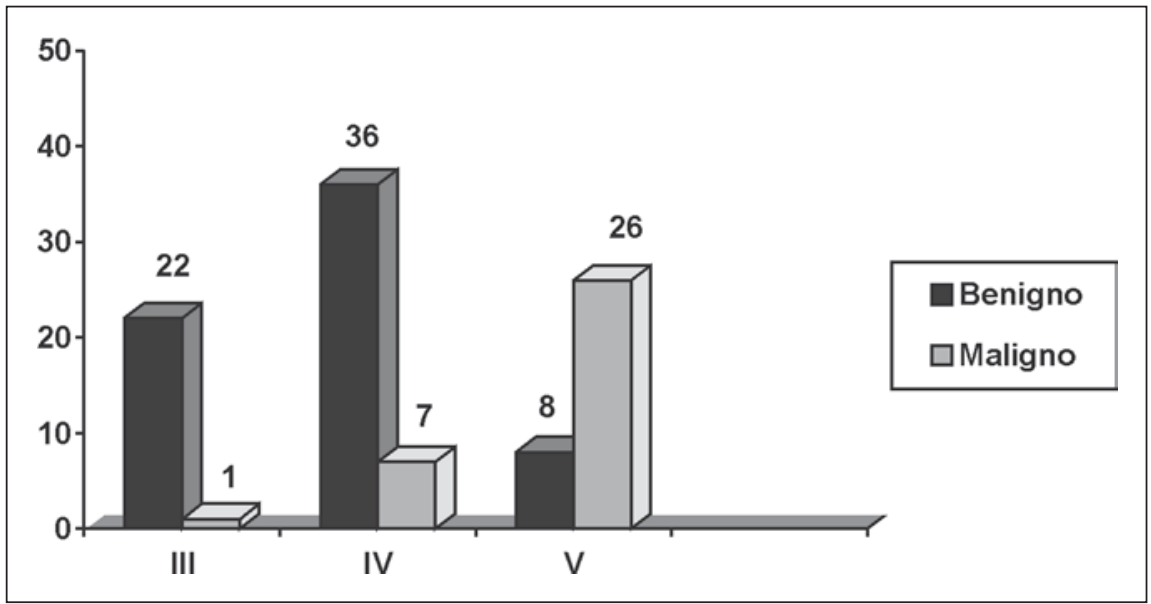

Gráfico 3. Relação entre o achado histopatológico e a categoria do BI-RADS.

permite o acesso mais fácil a todos os segmentos da mama. Ademais, este procedimento é realizado em tempo real, não há radiação e pode-se obter várias amostras em diferentes direções. No entanto, a lesão tem que ser ultra-sonograficamente identificada, o que não ocorre com as microcalcificações agrupadas sem nódulo associado e algumas massas sólidas podem não ser identificadas ao ultra-som.

Dentre as intervenções guiadas por ultra-som, a PBAAF tem sido amplamente utilizada para determinar a natureza da lesão mamária ${ }^{(1,2)}$. Apesar de ser minimamente invasiva e bem tolerada, tem inconvenientes: a análise da lesão é citológica, a obtenção de material insuficiente ocorre em grande porcentagem das amostras e, comumente, não se obtém a diferenciação entre carcinoma in situ e invasivo ${ }^{(3)}$. O diagnóstico negativo com a PBAAF fre- qüentemente não é clinicamente aceito como definitivo. É nesse contexto que a "core" biópsia vem ganhando espaço, uma vez que, comparando-a à PBAAF, apresenta como vantagem a análise histológica da lesão, avaliando fragmentos e proporcionando o diagnóstico diferencial entre as lesões benignas e malignas na maioria dos casos. Velez et al. ${ }^{(4)}$ demonstraram que as taxas de falso-negativo são significativamente menores em pacientes submetidas à "core" biópsia em comparação com as sujeitas à PBAAF (3,6\% versus $20 \%)$. Apesar de a PBAAF guiada por ultra-som ser útil para diferenciar os cistos complexos das lesões sólidas, ela tem menor acurácia em diferenciar lesão maligna de benigna. Os achados de Parker também suportam a afirmativa de que a "core" biópsia proporciona diagnóstico mais específico das lesões se comparada à $\mathrm{PBAAF}^{(5)}$. 
Além disso, a "core" biópsia é uma intervenção útil para diagnóstico tecidual de lesões mamárias suspeitas de malignidade (BI-RADS IV), lesões altamente suspeitas de malignidade (BI-RADS V) e em casos selecionados de lesões provavelmente benignas (BI-RADS III) que, por alguma razão, necessitam de investigação. Com relação ao custo/benefício, ao se comparar a "core" com a biópsia cirúrgica, Liberman et al. ${ }^{(6)}$ encontraram $77 \%$ de redução de procedimentos cirúrgicos com a "core" biópsia guiada por estereotaxia, diminuindo o custo do diagnóstico em 50\%. A biópsia percutânea ("core") é menos invasiva que a cirúrgica, remove menos tecido, causa menos deformidade mamária, não produz distorção nas mamografias subseqüentes e é realizada mais rapidamente.

No presente estudo, a indicação mais comum para o uso da "core" biópsia correspondeu à categoria IV (43\% das lesões). Dessas lesões, $28(65,1 \%)$ tiveram a "core" como resultado definitivo e $15(34,9 \%)$ foram submetidas à cirurgia, apresentando as seguintes indicações: cancerofobia (cinco casos), tratamento de doença maligna (oito casos), uma lesão por hiperplasia ductal atípica que teve diagnóstico anatomopatológico benigno após a cirurgia, e outra por diagnóstico de esclerose radial, cuja biópsia cirúrgica também foi benigna.

Em algumas situações a excisão cirúrgica é indicada após um diagnóstico benigno pela "core". Entre esses casos está a esclerose radial ou lesão esclerosante radial, que pode estar associada ao carcinoma, mais frequientemente ao tubular, adjacente à área em questão ou ao lobular ${ }^{(7)}$. A frequiência de carcinoma, à cirurgia, dessas lesões encontradas na "core" foi de $33 \%$ (duas de seis) na série de Jackman et $a l .{ }^{(\mathbf{8})}$ e de $25 \%$ (uma de quatro) na série de Lee et $a l .{ }^{(9)}$. Em nosso estudo, os casos com diagnóstico histológico com a "core" de esclerose radial não tiveram correlação com o carcinoma, tanto no grupo de categoria IV quanto no de categoria V.

Entre as outras situações em que após a "core" se indica a biópsia excisional, citam-se: hiperplasia ductal atípica, para diferenciação com o carcinoma in situ; na diferenciação do carcinoma in situ do invasivo e nos casos de discordância mamográfico-anatomopatológica. Em um estudo realizado por Dershaw et al. ${ }^{(\mathbf{1 0 )}}$, o carcinoma foi identificado em $15(50 \%)$ de 30 mulheres com diagnóstico de hiperplasia ductal atípica e em sete (47\%) de 15 mulheres com discordância mamográfico-patológica. Dos diagnósticos de carcinoma in situ pela "core", aproximadamente $20 \%$ têm áreas de invasão na análise da peça cirúrgica $^{(\mathbf{1 1 , 1 2})}$. Nessa situação, a mamotomia é de grande valia, pois o número de diagnósticos de hiperplasia ductal com atipia é bem menor.

Já em mulheres com lesões altamente suspeitas de malignidade (categoria $\mathrm{V}$ do BI-RADS) esse procedimento é útil quando substitui a biópsia cirúrgica, proporcionando um procedimento em tempo cirúrgico único. Isto facilita a discussão das opções cirúrgicas entre o médico e a paciente e a definição pré-operatória da técnica cirúrgica.

No presente estudo, em 34 casos a "core" foi realizada para a análise de lesões altamente suspeitas de malignidade (categoria $\mathrm{V}$ do BI-RADS). Desses, 26 pacientes $(58,8 \%)$ com diagnóstico de malignidade pela "core" tiveram o carcinoma mamário confirmado, sendo o tratamento cirúrgico o próximo passo. Acresce-se ainda que oito pacientes $(17,6 \%)$ não necessitaram de cirurgia, em virtude da eficiência do diagnóstico definitivo realizado pela "core", mas a fizeram por opção do cirurgião ou próprio desejo da paciente.

Uma outra vantagem desse método é o estudo das lesões multicêntricas. No presente estudo, por exemplo, uma mesma paciente apresentou duas alterações mamográficas na mesma mama, porém em quadrantes diferentes: uma na categoria III, outra na V; a "core" detectou carcinoma colóide na primeira e ductal invasivo na segunda, dados confirmados após análise histológica da peça cirúrgica.

Em relação às lesões mamárias sugestivas de benignidade (categoria III do BIRADS), estas podem ser seguidas mamograficamente. No entanto, se há suspeita de que a paciente não vai retornar para seguimento, ou quando esta solicita amostragem da lesão, a "core" biópsia pode ser um meio para evitar a cirurgia. Neste estudo, de 100 lesões submetidas à "core", 23 estão incluídas na categoria III (Gráfico 1). Apenas quatro lesões $(17,4 \%)$ foram sub- metidas a outro procedimento cirúrgico: por cancerofobia, por diagnóstico de carcinoma, por apresentação de aumento da lesão acompanhada de história familiar positiva para câncer de mama e, em outra, a "core" deixou dúvida entre fibroadenoma celular e tumor filóides. Isto porque em algumas lesões fibroepiteliais a amostra pela "core" pode ser insuficiente para distinção entre fibroadenoma e tumor filóides pelos patologistas $^{(\mathbf{1 0 )}}$.

A recomendação de excisão cirúrgica ocorre, ainda, frente ao diagnóstico de lesões papilares benignas ${ }^{(\mathbf{1 2})}$, mas há poucas referências no tocante a esta limitação. Em um estudo realizado por Rubin et al. ${ }^{(\mathbf{1 2})}$, sete lesões foram submetidas a biópsia cirúrgica após diagnóstico de lesão papilar pela "core" e todas apresentaram o mesmo diagnóstico após o ato cirúrgico.

As evidências dos achados concordantes entre a "core" biópsia e a biópsia cirúrgica foram, também, demonstradas em diversos estudos, como o de Parker et al. ${ }^{(13)}$, que descreveram 181 lesões biopsiadas com agulha 14 e pistola automática, guiadas por ultra-som, com quatro a cinco amostras por lesão. Em 49 lesões submetidas à biópsia cirúrgica, após a "core", houve $100 \%$ de concordância entre os achados anatomopatológicos da "core" e os da cirurgia. Nas outras 132 lesões benignas não foi identificado carcinoma após 12 a 36 meses de seguimento. Pijnappel et $a l .{ }^{(14)}$ também encontraram $100 \%$ de concordância dos achados histopatológicos entre a "core" e os espécimes cirúrgicos de lesões palpáveis, mas no grupo de lesões não-palpáveis, oito delas $(9,5 \%)$ não tiveram a mesma correlação. Destas, uma não teve amostra representativa da lesão e em outra encontraram-se hiperplasia ductal atípica no diagnóstico pela "core" e carcinoma ductal in situ no espécime cirúrgico. Ainda nesse estudo de Pijnappel et al., três casos de doença fibrocística não tiveram hiperplasia ductal atípica diagnosticada na "core", e outras três lesões, com carcinoma ductal in situ na "core", tinham áreas de invasão na peça cirúrgica.

Na casuística do presente estudo houve concordância entre os achados da "core" e os da biópsia excisional em 46 de 47 casos; em um caso (categoria V) o resultado da "core" foi hiperplasia ductal 
com atipia, e após análise da peça foi encontrado carcinoma ductal in situ. Este foi o único caso falso-negativo. Mas este achado histológico pela "core" já indica cirurgia em $100 \%$ dos casos, devido à alta correlação (em torno de 50\%) entre ele e o diagnóstico de carcinoma após a biópsia excisional, o que ocorre em uma freqüência bem menor na mamotomia.

Além disso, achados falso-positivos podem ocorrer em casos de adenose esclerosante e proliferação ductal esclerosante, em que uma mistura de proliferação epitelial e estroma nos tumores comumente simula a aparência do carcinoma invasivo.

Quanto às complicações decorrentes da "core", pode-se afirmar que são raras. A frequiência de hematoma e de infecção é, cada uma, menor que $1: 1.000^{(\mathbf{1 5})}$. Outras complicações possíveis são: hipersensibilidade, pneumotórax e disseminação carcinomatosa. Esta última é muito questionada devido ao fato de que a viabilidade das células é desconhecida e a disseminação de células malignas após biópsia percutânea de pulmão e órgãos abdominais, realizada há vários anos, não tem sido um problema $^{(\mathbf{1 6})}$. Nesta casuística não houve complicações após os procedimentos.

\section{CONCLUSÕES}

Este estudo pôde demonstrar que a "core" biópsia guiada pelo ultra-som é segura, confiável e menos traumática que a excisional, além de ser capaz de fornecer diagnóstico definitivo nas lesões sugestivas de benignidade e de malignidade. Propor- ciona, ainda, um único tempo cirúrgico para as lesões malignas, agilizando e facilitando a programação pré-operatória da cirurgia a ser realizada.

Foram descritas 47 lesões submetidas à cirurgia, dentre as quais se diagnosticaram 34 carcinomas, de modo que essas pacientes obtiveram resolução definitiva do caso após serem submetidas a um único procedimento cirúrgico.

Em nosso estudo evitou-se a biópsia excisional em 53 pacientes, preservandoas de uma cicatriz que pode proporcionar uma sensação de mutilação por parte da mulher. Essas pacientes tiveram um diagnóstico seguro através da "core", que é, como já mencionado, um método seguro, confiável e praticamente isento de cicatriz.

Entretanto, como toda técnica diagnóstica, é importante reconhecer suas limitações. Em particular, faz-se necessária investigação complementar com a biópsia cirúrgica, quando os diagnósticos de cicatriz radial, de hiperplasia ductal com atipia e de carcinoma ductal in situ sem invasão são feitos pela "core". Ênfase também deve ser dada à discordância entre o achado clínico, a imagem e o resultado da "core", para os quais a complementação do estudo histológico deve ser lembrada.

\section{REFERÊNCIAS}

1. Ciatto S, Rosselli Del Turco M, Bravetti P. Nonpalpable breast lesions: stereotaxic fine-needle aspiration cytology. Radiology 1989;173:57-9.

2. Gordon PB, Goldenberg SL, Chan NHL. Solid breast lesions: diagnosis with US-guided fineneedle aspiration biopsy. Radiology 1993;189: 573-80.
3. Dershaw DD. Percutaneous biopsy of nonpalpable breast lesions: core or fine needle aspiration. In: Dershaw DD, ed. Interventional breast procedures. New York: Churchill Livingstone, 1995:103-6.

4. Velez N, Earnest DE, Staren ED. Diagnostic and interventional ultrasound for breast disease. Am J Surg 2000;180:284-7.

5. Parker SH. Percutaneous large core breast biopsy. Cancer 1994:74(1 Suppl):256-62.

6. Liberman L, Dershaw DD, Rosen PP, et al. Stereotaxic core biopsy of breast carcinoma: accuracy at predicting invasion. Radiology 1995;194:379-81.

7. Rosen PP. Radial sclerosing lesions. In: Rosen PP, ed. Rosen's breast pathology. Philadelphia: Lippincott-Raven, 1997:76-81.

8. Jackman RJ, Finkelstein SI, Marzoni FA. Stereotaxic large-core needle biopsy of histologically benign nonpalpable breast lesions: false-negative results and failed follow-up. Radiology 1995:197 (P):(abstr)

9. Lee CH, Egglin TK, Philpotts LE, Mainiero MB, Tocino I. Cost-effectiveness of stereotactic core needle biopsy: analysis by means of mammographic findings. Radiology 1997;202:849-54.

10. Dershaw DD, Morris EA, Liberman L, Abramson AF. Nondiagnostic stereotaxic core breast biopsy: results of rebiopsy. Radiology 1996;198:323-5.

11. Jackman RJ, Nowels KW, Shepard MJ, Finkelstein SI, Marzoni FA Jr. Stereotaxic large-core needle biopsy of 450 nonpalpable breast lesions with surgical correlation in lesions with cancer or atypical hyperplasia. Radiology 1994;193:91-5.

12. Rubin E, Dempsey PJ, Pile NS, et al. Needle-localization biopsy of the breast: impact of a selective core needle biopsy program on yield. Radiology 1995;195:627-31.

13. Parker SH, Jobe WE, Dennis MA, et al. US-guided automated large-core breast biopsy. Radiology 1993;187:507-11.

14. Pijnappel RM, van Dalen A, Rinkes IH, van den Tweel JG, Mali WP. The diagnostic accuracy of core biopsy in palpable and non-palpable breast lesions. Eur J Radiol 1997;24:120-3.

15. Parker SH, Burbank F, Jackman RJ, et al. Percutaneous large-core breast biopsy: a multi-institutional study. Radiology 1994;193:359-64.

16. Parker SH, Burbank F. A practical approach to minimally invasive breast biopsy. Radiology 1996; 200:11-20. 